Research Report

\title{
International Consensus on the Clinical Management of Inflammatory Breast Cancer from the Morgan Welch Inflammatory Breast Cancer Research Program 10th Anniversary Conference
}

Naoto T. Ueno ${ }^{1 \bowtie *}$, Jose Rodrigo Espinosa Fernandez ${ }^{1 *}$, Massimo Cristofanilli2 ${ }^{2}$ Beth Overmoyer ${ }^{3}$, Dan Rea ${ }^{4}$, Fedor Berdichevski ${ }^{4}$, Mohamad El-Shinawi ${ }^{5}$, Jennifer Bellon ${ }^{6}$, Huong T. Le-Petross ${ }^{1}$, Anthony Lucci ${ }^{1}$, Gildy Babiera1, Sarah M. DeSnyder ${ }^{1}$, Mediget Teshome1, Edward Chang1, Bora Lim¹, Savitri Krishnamurthy1, Michael C Stauder ${ }^{1}$, Simrit Parmar 6 , Mona M Mohamed7, Angela Alexander ${ }^{1}$, Vicente Valero ${ }^{1}$, Wendy A. Woodward $1 \bowtie$

1. Morgan Welch Inflammatory Breast Cancer Research Program and Clinic, The University of Texas MD Anderson Cancer Center, Houston, Texas, USA

2. Department of Medicine, Division of Hematology and Oncology, Robert H Lurie Comprehensive Cancer Center, Northwestern University, Evanston, Illinois, USA

3. Susan F. Smith Center for Women's Cancers, Dana-Farber Cancer Institute, Boston, Massachusetts, USA

4. School of Cancer Sciences, University of Birmingham, Edgbaston, Birmingham, UK

5. Department of General Surgery, Faculty of Medicine, Ain Shams University, Cairo, Egypt

6. Department of Stem Cell Transplantation and Cellular Therapy, The University of Texas MD Anderson Cancer Center, Houston, Texas, USA

7. Department of Zoology, Faculty of Science, Cairo University, Giza, Egypt

${ }^{*}$ Equal first authors.

$\square$ Corresponding author: Naoto T. Ueno, M.D., Ph.D., F.A.C.P., Morgan Welch Inflammatory Breast Cancer Research Program and Clinic, Department of Breast Medical Oncology, Unit 1354, The University of Texas MD Anderson Cancer Center, 1515 Holcombe Blvd., Houston, Texas 77030. Phone: 713-792-8754; E-mail: nueno@mdanderson.org. Wendy A. Woodward, M.D., Ph.D., Associate Professor of Radiation Oncology, The University of Texas MD Anderson Cancer Center, Box 1202, 1515 Holcombe Blvd, Houston, TX 77030; Fax: (713) 563-6940; E-mail: wwoodward@mdanderson.org.

(c) Ivyspring International Publisher. This is an open access article distributed under the terms of the Creative Commons Attribution (CC BY-NC) license (https://creativecommons.org/licenses/by-nc/4.0/). See http://ivyspring.com/terms for full terms and conditions.

Received: 2017.11.21; Accepted: 2018.01.29; Published: 2018.04.06

\begin{abstract}
National and international experts in inflammatory breast cancer (IBC) from high-volume centers treating IBC recently convened at the 10th Anniversary Conference of the Morgan Welch Inflammatory Breast Cancer Research Program at The University of Texas MD Anderson Cancer Center in Houston Texas. A consensus on the clinical management of patients with IBC was discussed, summarized, and subsequently reviewed. All participants at the conference (patients, advocates, researchers, trainees, and clinicians) were queried using the MDRing electronic survey on key management issues. A summary of the expert consensus and participant voting is presented. Bilateral breast and nodal evaluation, breast magnetic resonance imaging, positron emission tomography/computed tomography, and medical photographs were endorsed as optimal. Neoadjuvant systemic therapy, modified radical mastectomy and level I and II ipsilateral axillary node dissection, post-mastectomy radiotherapy, adjuvant targeted therapy and hormonal therapy as indicated, and delayed reconstruction were agreed-upon fundamental premises of standard non-protocol-based treatment for IBC. Consideration for local-regional therapy in de novo stage IV IBC was endorsed to provide local control whenever feasible. Variation across centers and special circumstances were discussed.
\end{abstract}

Key words: inflammatory breast cancer, management, BCS, radiation, mastectomy, neoadjuvant chemotherapy. 


\section{Introduction}

In the past 10 years, coordinated focus on the treatment and biology of inflammatory breast cancer (IBC) has yielded significant progress in the clinical outcome. Incremental advances in systemic therapies, staging, surgery, new technology-based radiotherapy, treatment options for recurrence, standardized approach to a multidisciplinary management, and targeted therapy clinical trials based on IBC-specific data have improved outcomes over time.

Collaboration between The University of Texas MD Anderson Cancer Center (MDA) clinicians and Texas and New Mexico state legislators led to the formation of a dedicated IBC clinic at MDA in 2006. This ushered forward coordination of an IBC World Consortium of national and international experts and led to the development of formalized IBC centers at several other major centers. National and international experts in IBC from high-volume centers treating IBC recently convened at the 10th Anniversary Conference of the Morgan Welch Inflammatory Breast Cancer Research Program at MDA. Review of the MDA experience shows that the 5 -year actuarial stage III overall survival has increased from $40-50 \%$ (for patients treated in the 1970s, 1980s, and 1990 s) to $68 \%$ for patients treated since 2008 , as reported at the conference.

Herein, we review specific practices among high-volume IBC centers and practitioners, highlighting where there was a clear consensus and where experts agreed multiple approaches are reasonable given insufficient evidence for a single superior approach. For instances of significant dissenting views, the rationale and rebuttal were addressed. Overall, there was substantial consensus for recommendation of workup including bilateral breast and nodal evaluation, breast magnetic resonance imaging (MRI), positron emission tomography/computed tomography (PET/CT), and medical photographs. Neoadjuvant systemic therapy (NST), modified radical mastectomy and level I and II axillary nodal dissection, post-mastectomy radiotherapy to the chest wall and nodal basin, and delayed reconstruction were agreed-upon fundamental premises of standard non-protocol-based treatment for IBC. Consideration for local-regional therapy in de novo stage IV IBC was endorsed to provide local control whenever feasible.

\section{Participants}

Clinicians involved in the conference represented a broad cohort of IBC experts from high-volume centers. We include here a brief description of the programs represented.
Because MDA is a tertiary referral center, IBC patient volume is higher, which led to the creation in 2006 of the Morgan Welch Inflammatory Breast Cancer Research Program and Clinic, a dedicated program that sees over 100 new IBC cases per year. Similarly, the dedicated IBC program at the Susan F. Smith Center for Women's Cancers at the Dana-Farber Cancer Institute has provided IBC care in the northeastern United States since its creation by $\mathrm{Dr}$ Beth Overmoyer in 2009 and currently sees 60 to 80 patients per year. Dr Massimo Cristofanilli, at the conference representing the Robert $\mathrm{H}$. Lurie Comprehensive Cancer Center of Northwestern University, was integral to the inauguration of the MDA program and international consortium and has practiced in clinical IBC programs in multiple academic centers.

Throughout and after the conference, attendees, as well as other physicians who manage IBC, were surveyed using MDRing, a mobile and web based application that allows for users to answer multiple choice questions in real time (1-3). Through a simple question and answer format that included common clinical scenarios concerning IBC clinical management, practitioners were surveyed about the approach they use in daily practice. These various scenarios, as well as the answers to the survey, are described throughout the consensus. Work is ongoing to continue to identify and survey international IBC clinical leaders. All interested parties are welcome to contact the corresponding authors for further information.

\section{Staging workup in the setting of clinical IBC symptoms and a biopsy demonstrating invasive mammary carcinoma}

The panel strongly endorsed the value of upfront medical photography to document findings required for diagnosis and to inform all members of the multidisciplinary oncology team who may meet the patients only after resolution of skin findings. In some cases, patients may have taken these photos themselves if presenting after initiating systemic chemotherapy. Photos obtained by patients should be filed in the medical record.

A consensus was not reached on the best upfront diagnostic tool, and most of the experts agreed that in patients without a diagnosis, bilateral mammogram followed by bilateral breast and nodal basin ultrasound are the standard initial imaging modalities, owing to the relatively low cost and good accessibility outside of an academic institution (4). Relevant mammographic findings include skin thickening (84-93\%), trabecular thickening (62-81\%), trabecular distortion $(37 \%)$, increased breast density 
(93\%), calcifications (47-56\%), and axillary adenopathy $(24 \%)$, although mammography is the least sensitive method for primary breast lesion detection $(5,6)$. The utility of bilateral breast imaging to assess both bilateral breast cancer at presentation or cross lymphatic metastasis to the contralateral breast or nodal basin was described, owing to the prevalence of contralateral breast cancer in up to $5 \%$ of IBC patients at 2 years compared with approximately $1.1 \%$ at 2 years for non-IBC patients (7). Relevant ultrasound findings include skin thickening, a breast mass or diffuse abnormal non-mass hypoechoic tissue with posterior acoustic shadowing, hyperechoic foci representing calcifications, and nodal disease $(5,8,9)$. If a primary breast lesion (mass or non-mass lesion) can be identified by ultrasound, then a core biopsy of the primary breast lesion and fine needle aspiration or core biopsy of suspicious axillary or infra/supraclavicular lymph node(s) should be performed $(5,10)$.

Although data regarding the role of breast MRI and PET/CT for IBC specifically are lacking, retrospective evidence and the clinical utility of both modalities were reviewed. Breast MRI can detect a primary breast lesion in up to $100 \%$ of cases and the lesion can be targeted for core biopsy (11). MRI has also been described as the most sensitive study to diagnose multi-centric disease (12). Finally, MRI best demonstrates the extent of disease, including ipsilateral and contralateral skin involvement, enhancing skin lesions or diffuse thickening of the skin (97\%), and breast and/or chest wall edema.
Initial rapid enhancement with delayed washout or plateau curve is seen in most cases of IBC (97\%) (11, 13). A study comparing the vascular patterns in 57 IBC patients with those of 54 non-IBC patients demonstrated a significant difference in the pattern of angiogenesis. More dome configuration was observed in IBC than in non-IBC (64\% compared with $42 \%, \mathrm{p}=$ 0.03 ) and higher pre-pectoral vascular clusters appeared in IBC than in non-IBC (75\% compared with $41 \%, \mathrm{p}=0.001)$. These findings suggest that the inflammatory component may trigger increased vascularity and unique vascular characteristics in IBC (H.T. Le-Petross, unpublished data).

The role of PET/CT in the staging of breast cancer, especially aggressive forms such as IBC, has not been clearly established. PET/CT can show not only the primary breast tumors as hyper metabolic lesions but also nodal involvement ( $90 \%$ of patients) and unsuspected distant metastase in up to $49 \%$ of IBC patients $(14,15)$. Retrospective data have shown that, unlike in the non-IBC locally advanced cancer setting, for patients with stage III IBC, adding PET/CT to staging on the basis of conventional imaging could detect metastases not detected by conventional imaging, thereby having a statistically significant effect on recurrence-free survival ( $p=$ 0.014) (16). These are retrospective findings that need to be confirmed prospectively. Owing to the aggressive behavior of $\mathrm{IBC}$, however, experts tend to recommend PET/CT as a staging workup tool; although a bone scan and CT studies of the chest, abdomen, and pelvis could serve as an alternative.

Table 1: Optimal workup for inflammatory breast cancer

\begin{tabular}{|c|c|}
\hline Modality & Rationale and caveats \\
\hline Medical photography & $\begin{array}{l}\text { Medical photographs and examinations prior to neoadjuvant chemotherapy are critical to visually determine the } \\
\text { extent of skin involvement relative to future radiotherapy fields and surgery planning. }\end{array}$ \\
\hline Bilateral mammogram & For detection of microcalcifications and contralateral disease. Global skin thickening can be seen. \\
\hline Ultrasound & For performing image-guided biopsy of a primary breast lesion and for nodal staging. \\
\hline Breast magnetic resonance imaging & $\begin{array}{l}\text { For detecting a primary breast lesion (mass or non-mass enhancement), skin thickening, breast and chest wall } \\
\text { edema, chest wall and nodal involvement and contralateral breast assessment. }\end{array}$ \\
\hline $\begin{array}{l}\text { Positron emission tomography/computed } \\
\text { tomography (PET/CT) }\end{array}$ & $\begin{array}{l}\text { For detecting distant metastatic disease and assessing local-regional disease extent, aiding radiation and surgical } \\
\text { planning. }\end{array}$ \\
\hline $\begin{array}{l}\text { Bone scan and CT of the chest, abdomen, } \\
\text { and pelvis }\end{array}$ & $\begin{array}{l}\text { Standard staging workup for metastatic bone disease if PET/CT is not performed. If PET/CT is not available, } \\
\text { combined bone scan with standard CT scans (chest, abdomen, and pelvis). }\end{array}$ \\
\hline Pathology & $\begin{array}{l}\text { Core needle biopsy and skin punch biopsy for standard histopathologic examination to establish a diagnosis of } \\
\text { invasive mammary carcinoma and for biomarker evaluation. }\end{array}$ \\
\hline
\end{tabular}

Table 2: MDRing responses for inflammatory breast cancer workup

\begin{tabular}{|c|c|c|}
\hline \multirow[t]{2}{*}{ Question } & \multicolumn{2}{|c|}{ Answers } \\
\hline & Yes & No \\
\hline Magnetic resonance imaging is indicated for all patients with newly diagnosed inflammatory breast cancer & $66 \%$ & $34 \%$ \\
\hline Positron emission tomography/computed tomography is appropriate for all patients with newly diagnosed inflammatory breast cancer & $71 \%$ & $29 \%$ \\
\hline
\end{tabular}


Finally, the importance of pre-systemic therapy cross-sectional imaging in cases of regional nodal involvement was highlighted, noting that the precision of radiotherapy relies on an understanding of the specific anatomic distribution of disease upfront (or prior to systemic therapy). Pre-treatment PET/CT imaging has also provided information on the extent of local disease resulting in modifications in post-mastectomy radiation treatment fields and radiation doses. Studies highlighting the role of PET/CT to change stage, surgery, or radiotherapy fields were reviewed (16-18). A few experts opined that in patients with classic symptoms of IBC, mammography does not add relevant clinical information. A proposed algorithm to be clinically validated in the future for clinical suspicion of IBC is breast MRI (to identify the primary breast lesion for ultrasound-guided biopsy and to detect skin lesions or skin enhancement suggesting tumor emboli in skin), ultrasound after MRI (to biopsy the most likely primary lesion detected on MRI and for locoregional nodal staging with possible nodal biopsy), and PET/CT (for local and distant disease workup).

\section{Pathology}

The role of pathology in the diagnosis, management, and clinical trial development of IBC was discussed. Because IBC is a clinicopathologic entity, pathologic confirmation of invasive breast carcinoma using image-guided core needle biopsy (CNB) of the affected breast is an essential part of diagnosis (19). Skin punch biopsy is recommended for examination of invasive carcinoma and dermal tumor emboli, with two 2-mm to 8-mm skin punch samples from the most prominent area of breast skin discoloration, however not mandated to make the diagnosis.

Lymphovascular tumor emboli is a frequent feature of IBC, demonstrated in skin punch biopsy in up to $75 \%$ of patients, but their absence does not rule out the diagnosis (20).

IBC is generally ductal in origin. However, other histological variants of mammary carcinoma can be encountered. The histologic characteristics of the invasive tumor do not appear to influence the survival in patients with IBC, thereby indicating that IBC is a distinct clinicopathologic entity that transcends histologic distinction (21).

A hallmark of the invasive tumor and lymphovascular tumor emboli in IBC is the overexpression of E-cadherin, a calcium-dependent transmembrane glycoprotein responsible for cell adhesion. An overactive E-cadherin beta-catenin axis is suggested to be critical for the genesis of lymphovascular tumor emboli in IBC (22). IBC can belong to any of the currently known intrinsic subtypes of breast cancer. Pathologic evaluation of mastectomy specimens following completion of NST entails a detailed and extensive sampling of the breast parenchyma and skin, to determine the extent of residual tumor burden for accurate final pathologic staging of the patient.

It is important to highlight the challenges of tissue procurement for undertaking correlative studies that are designed as an integral part of clinical trials for patients with IBC. The unique pathologic characteristics of the invasive tumor that are intrinsic to IBC, including noncontiguous areas of infiltration, reduced density of tumor cells, and edema of stroma separating the clusters of tumor cells, are features that pose significant problems in procuring high-quality pre-treatment core biopsies from patients with IBC. The quality of procured tissue is proportionate to the needle gauge used, length of the core, cellularity of the invasive tumor, and density of cells. The tumor cellularity of the procured core needle biopsy (CNB), is unpredictable regardless of whether they are fixed in formalin, snap frozen, embedded in optimal cutting temperature solution, or kept in RNA Later solution (Ambion) for designed genomic and proteomic analysis. The reported guidelines for collecting CNBs in clinical trials do not ensure collection of high-quality tissue (23). Our inability to assess the tumor cellularity of CNBs at the time of procurement is indeed a major limitation that can preclude successful completion of the correlative studies that are planned in the clinical trial. There is a need for better assessment of specimen tumor cellularity at the time of procurement.

Optical imaging modalities that can allow visualization of the tissue at the bedside without the need for tissue processing are emerging. These modalities hold promise for immediate estimation of the quality of the CNB, thereby allowing optimal collection of tissue at the time of procurement. The results from a feasibility study, evaluating the use of confocal fluorescence microscopy for rapid evaluation of tumor cellularity in CNBs obtained from patients with IBC, needs further validation before it can be incorporated into routine clinical practice (24). The current availability of high-throughput genomic testing platforms enables us to obtain genomic data provided the required amount of nucleic acids is extracted from the collected tissue. The current practice of using single formalin-fixed and paraffin-embedded tissue sections for the proposed protein biomarkers to be estimated by immunohistochemistry can exhaust the tissue and limit the number of biomarkers that can be estimated. The currently available multiplexing platform for 
evaluating multiple biomarkers is investigational and needs validation for standard evaluation of multiple biomarkers from a single tissue section. There is a great need for high-throughput proteomic platforms that will enable evaluation of multiple protein biomarkers from limited tissue.

\section{Systemic therapy}

All participants agreed that upfront primary systemic therapy, including chemotherapy or chemotherapy plus targeted therapy, is indicated in patients with stage III IBC. In patients with stage IV de novo IBC, participants also recommended primary systemic therapy to achieve optimal response, and patients should be evaluated for surgery and radiation therapy using a multidisciplinary approach. Although the impact of surgery and radiation therapy in terms of time to progression and overall survival are controversial in this setting, the morbidity of loco-regional progression is obvious and merits multidisciplinary intervention in those patients who have significant clinical response to systemic therapy, are well enough to undergo intensive local-regional therapy and have a life expectancy long enough to expect local progression to be an issue.

Standard systemic therapy regimens used off protocol across institutions included multiple national guideline-concordant approaches, and such variation reflects the absence of data demonstrating one superior regimen for IBC.

All experts agreed that sequential treatment with doxorubicin and cyclophosphamide, then a taxane with or without carboplatin should be administered prior to surgery in patients with HER2-negative disease.

The backbone for primary chemotherapy is regimens that include an anthracycline and taxanes. This recommendation is mainly based on extrapolation of data available from prospective trials evaluating non-IBC patients. Some of these trials have included analyses specifically in IBC populations, with inconsistent results in terms of clinical outcome between IBC and non-IBC populations (25-27). It was suggested that the clinical response of non-IBC is not an appropriate surrogate for IBC. Therefore, the inclusion of IBC patients in clinical trials for the treatment of locally advanced breast cancer can dilute the interpretation of results and dedicated clinical trials investigating new strategies specifically for IBC are urgently needed.

The largest study specifically analyzing IBC patients who received an anthracycline-based regimen followed by locoregional therapy reported a 10 -year overall survival rate of $33 \%(28)$, so the recommendation of MDA is the use of upfront anthracycline-based therapy. With the objective of raising $\mathrm{pCR}$ rates, Dana Farber Cancer Institute has incorporated a dose-dense anthracycline and taxane regimen for high-risk patients (25). Therefore, the recommendation was that patients receive taxane and doxorubicin and cyclophosphamide for primary IBC, to achieve the highest $\mathrm{pCR}$ rate.

For HER2-positive disease, all institutions and participants agreed that dual anti-HER2-directed therapy with pertuzumab and trastuzumab should be used. In the NOAH trial, a pivotal trial that demonstrated the benefit of the anti-HER2 agent trastuzumab in the neoadjuvant setting, $20 \%$ of patients had IBC. A subgroup analysis in this population showed that trastuzumab added to chemotherapy increased the $\mathrm{pCR}$ rate to $48 \%$, translating into an improvement of 5-year event-free survival (64\% compared with $24 \%$, hazard ratio 0.34 ) and 5-year overall survival (74\% compared with $44 \%$, hazard ratio 0.38) specifically in IBC patients (29). Data was presented supporting paclitaxel (T), pertuzumab $(\mathrm{P})$ and trastuzumab $(\mathrm{H})$ as preoperative systemic therapy for HER2-positive cancers (Overmoyer et al., San Antonio Breast Cancer Symposium 2017, abstract \#501; manuscript in process). Dual anti-HER2 blockade was studied in the NeoSphere trial, in which $7 \%$ of patients had IBC, and the combination of pertuzumab and trastuzumab with chemotherapy raised the pCR rate to $45.8 \%$ (95\% confidence interval 36.1-55.7) (30). Therefore, all attendees agreed to consider dual anti-HER2 therapy (pertuzumab and trastuzumab) combined with chemotherapy as the first option, with the objective of achieving superior $\mathrm{pCR}$ rates.

A non-anthracycline-containing regimen, consisting of a taxane, carboplatin, trastuzumab, and pertuzumab (TCHP) was recommended by some of the participants, based on the TRYPHAENA trial (in which $6 \%$ of patients had IBC, and carboplatin raised the pCR rate to $64 \%$ ) (31). Because efficacy was not the primary objective of the TRYPHAENA trial, there was not enough evidence for the substitution of anthracyclines with carboplatin, so a consensus was not achieved. Most participants agreed that the carboplatin-containing regimen should be carefully selected owing to toxicity considerations. Therefore, participants agreed that for HER2-poisitve cancers, doxorubicin and cyclophosphamide (AC) $\quad x \quad 4$ followed by TPH is the standard regimen for primary HER2 positive IBC to achieve the highest $\mathrm{pCR}$ rate.

In a discussion of adjuvant therapy for HER2-positive disease, MDA and Dana-Farber Cancer Institute agreed that the standard 1-year anti-HER2 therapy should be trastuzumab and pertuzumab. This recommendation now based on the 
recently published results from the APHINITY trial that showed a modest reduction in disease recurrence events $(7.1 \%$ compared with $8.7 \%$, hazard ratio 0.81 ; p $=0.045)$, with the addition of pertuzumab to taxane/trastuzumab containing regimen in the adjuvant setting. This effect is most detectable among patients with an increased risk of disease relapse (i.e., lymph node involvement or hormone receptor negativity) (32). Although this trial was not done specifically in the IBC patient population, the high relapse rate observed in IBC patients and major benefit observed in high-risk patients with breast cancer made participants lean toward recommending this intervention. Through the question and answer (MDRing) system provided during the meeting, 73\% of experts attending the conference responded favorably to considering adjuvant dual HER2-directed therapy for 1 year.

For estrogen receptor-positive disease, all participating institutions agreed that tamoxifen should be administered for 10 years in premenopausal women and aromatase inhibitors should be administered for 5 years in postmenopausal women. Based upon the SOFT trial, ovarian suppression is recommended for premenopausal women with hormone receptor positive IBC (33) due to the benefit in DFS, as long as quality of life is acceptable and long-term side effects are well explained to the patients. Whether to use aromatase inhibitors or tamoxifen with ovarian suppression as adjuvant endocrine therapy in premenopausal women was debated without consensus.

No consensus emerged on the use of carboplatin for triple-negative IBC. Representatives of both MDA and Dana-Farber Cancer Institute agreed that although neoadjuvant carboplatin has been shown to increase $\mathrm{pCR}$ rates in triple-negative breast cancer (34, $35)$, there is still not enough evidence supporting long term clinical efficacy to justify its routine use. In addition, the currently available trial data have excluded IBC patients or do not show specific results for this patient population. Therefore, a consensus did not emerge and most participants did not recommend adding carboplatin to taxane therapy outside of a clinical trial.

In patients with residual invasive disease after NST, the addition of six to eight cycles of capecitabine to standard adjuvant therapy was discussed, on the basis of results of the CREATE-X study showing an increase in disease-free and overall survival, in HER2-negative breast cancer patients with known unfavorable outcomes (36). However, this was not agreed upon all experts, and the recommendation by some participants was to consider adding capecitabine to adjuvant treatment, for triple negative IBC patients who do not achieve a pCR.

For stage IV disease, no consensus emerged on a preferred treatment regimen and all institutions agreed on the use of their own standard chemotherapy regimens for locally advanced disease. All participants agreed that the best-response chemotherapy regimen should be used, with the objective of reaching a window of opportunity for local control, taking into consideration patient performance status and quality of life.

Finally, owing to the clear lack of randomized prospective controlled trials specifically in the IBC patient population, a recommendation was made to encourage care givers and patients to participate in clinical trials specifically designed for IBC patients, which can yield level I evidence that can support clinical care decisions.

Table 3. Neoadjuvant and adjuvant systemic therapy regimens endorsed across centers

\begin{tabular}{|c|c|c|c|c|}
\hline \multirow[t]{2}{*}{ Institution } & \multicolumn{2}{|l|}{ Neoadjuvant chemotherapy } & \multirow[t]{2}{*}{ Comments } & \multirow[t]{2}{*}{ Adjuvant therapy } \\
\hline & HER2-positive & HER2-negative & & \\
\hline MD Anderson & AC $\times 4$ followed by THP & $\begin{array}{l}\text { AC } \times 4 \text { followed by } \\
\text { paclitaxel weekly } \\
\text { (or the opposite } \\
\text { sequence) }\end{array}$ & $\begin{array}{l}\text { Anthracycline therapy upfront; no } \\
\text { evidence for carboplatin in } \\
\text { triple-negative disease outside of a } \\
\text { clinical trial }\end{array}$ & $\begin{array}{l}\text { HER2-positive: trastuzumab } \times 1 \text { year (refer to text } \\
\text { for data concerning adjuvant pertuzumab); } \\
\text { ER-positive: tamoxifen or LHRH/AI for } \\
\text { premenopausal and AI for postmenopausal; no } \\
\text { standard systemic chemotherapies }\end{array}$ \\
\hline $\begin{array}{l}\text { Dana-Farber } \\
\text { Cancer Institute }\end{array}$ & $\begin{array}{l}\text { THP weekly followed by; } \\
\text { AC } \times 4 \text { (either neoadjuvant } \\
\text { or adjuvant)* }\end{array}$ & $\begin{array}{l}\text { Dose-dense AC } \\
\text { followed by } \\
\text { dose-dense } \\
\text { paclitaxel }\end{array}$ & $\begin{array}{l}\text { *All patients receive AC after surgical } \\
\text { treatment; for those with a poor } \\
\text { response to TPH, AC is administered } \\
\text { before surgery; no evidence for } \\
\text { carboplatin in triple-negative disease } \\
\text { outside of a clinical trial }\end{array}$ & $\begin{array}{l}\text { HER2-positive: Trasztuzumab and Pertuzumab to } \\
\text { complete } 12 \text { mo therapy; ER-positive: LHRH/AI for } \\
\text { premenopausal and AI for postmenopausal; } \\
\text { consider adding capecitabine to adjuvant treatment } \\
\text { for triple negative IBC patients who do not achieve } \\
\text { a pCR }\end{array}$ \\
\hline $\begin{array}{l}\text { Northwestern } \\
\text { University }\end{array}$ & $\begin{array}{l}\text { Paclitaxel and trastuzumab } \\
\text { weekly; AC } \times 4\end{array}$ & AC; paclitaxel & & \\
\hline
\end{tabular}


Table 4: MDRing responses for systemic therapy in inflammatory breast cancer (IBC)

\begin{tabular}{|c|c|}
\hline Question & Answers \\
\hline $\begin{array}{l}\text { A } 39 \text {-year-old premenopausal patient with ER-positive, PR-negative, and HER2-negative IBC has an } \\
\text { estradiol level of } 95 \mathrm{pg} / \mathrm{mL} \text { after completing systemic chemotherapy and locoregional therapy. What is } \\
\text { the standard adjuvant hormonal therapy? }\end{array}$ & $\begin{array}{l}62 \% \text { answered luteinizing hormone-releasing hormone } \\
\text { agonist plus aromatase inhibitor } \\
38 \% \text { answered tamoxifen }\end{array}$ \\
\hline $\begin{array}{l}\text { A } 45 \text {-year-old patient with triple-negative IBC received four cycles of dose-dense doxorubicin and } \\
\text { cyclophosphamide } \times 4 \text { followed by four cycles of paclitaxel and carboplatin. After surgery she has } \\
\text { residual multifocal disease (largest diameter } 2.5 \mathrm{~cm} \text { ) two of } 16 \text { axillary lymph nodes are positive for } \\
\text { metastasis. Would you consider adjuvant systemic chemotherapy with six cycles of capecitabine? }\end{array}$ & $\begin{array}{l}80 \% \text { answered yes } \\
20 \% \text { answered no }\end{array}$ \\
\hline In a patient with IBC that has progressed after systemic therapy, the best next step is... & $\begin{array}{l}3 \% \text { answered definite radiotherapy without surgery } \\
3 \% \text { answered immediate mastectomy } \\
84 \% \text { answered new systemic therapy with a plan for } \\
\text { mastectomy if further progression occurs and threatens } \\
\text { the operable window } \\
10 \% \text { answered preoperative radiotherapy }\end{array}$ \\
\hline Is carboplatin a standard chemotherapy component in triple-negative stage III IBC? & $\begin{array}{l}46 \% \text { answered yes } \\
54 \% \text { answered no }\end{array}$ \\
\hline $\begin{array}{l}\text { Is comprehensive locoregional therapy, modified radical mastectomy, plus axillary lymph node } \\
\text { dissection and post-mastectomy radiotherapy to the chest wall and nodal basin appropriate in patients } \\
\text { with de novo stage IV IBC that is improving after chemotherapy with or without targeted therapy? }\end{array}$ & $\begin{array}{l}82 \% \text { answered yes } \\
18 \% \text { answered no }\end{array}$ \\
\hline $\begin{array}{l}\text { Is the combination of pertuzumab and trastuzumab for a } 1 \text { year a standard targeted therapy for stage } \\
\text { III HER } 2+\text { IBC? }\end{array}$ & $\begin{array}{l}54 \% \text { answered yes } \\
46 \% \text { answered no }\end{array}$ \\
\hline
\end{tabular}

Abbreviations: ER, estrogen receptor; PR, progesterone receptor.

\section{Surgery}

There was widespread but not unanimous consensus that modified radical mastectomy and level I and II axillary node dissection with delayed reconstruction is the only guideline-concordant surgical option for IBC. A critical concern is not to leave disease behind; thus, achieving negative margins and removing involved skin are considered standard. Skin-sparing mastectomy and breast-conserving surgery (BCS) have not been prospectively studied in IBC patients. Breast-conserving therapy is largely considered contraindicated for patients with IBC in national guidelines because these patients were not included in randomized trials showing equivalence of breast conservation and radiation therapy compared to mastectomy. Local progression in IBC can be a serious problem and at worst lead to carcinoma en cuirasse with very extensive invasion that may be painful, ulcerate, bleed and become infected and is distressing for patients and care-givers. Even in patients with a high risk of competing distant metastasis, an expectation of improved rate of local control benefit in and of itself is considered by many a compelling reason to recommend mastectomy. Developing trials in early stage breast cancer are testing whether pathologic complete response may predict for those who can undergo less surgery however these have not been extended to include locally advanced patients. Indeed, the only prospective data regarding less surgery in complete responders with locally advanced breast cancer including IBC demonstrated higher local recurrence among those without complete response who had surgery than among those without surgery who achieved a pathologic complete response (37). Mastectomy is therefore recommended regardless of response in operable patients. Chest wall coverage with reconstructive techniques (i.e., latissimus dorsi flap) may be utilized when extensive skin resection is necessary given the extent of disease. A further practice point to emphasize here is that patients who experience disease progression during systemic therapy require particularly close monitoring with timely transition to surgery if progression to an inoperable status is imminent. Only when this operable window has closed is preoperative radiotherapy or chemoradiation offered.

It was noted that the recent United Kingdom guidelines stop short of recommending mastectomy in all cases, on the basis of retrospective review of national practice that suggested outcomes were favorable in the subset of IBC patients who received BCS (38). A recent publication from the Edinburgh Breast Unit in the United Kingdom advocated BCS in selected patients with IBC, after reviewing 35 patients from a prospectively collected database over a 14-year period. Over a long follow-up period (median 80 months), excellent results were demonstrated in locoregional recurrence-free survival $(87.5 \%$ at 5 years) and actuarial survival (70.3\% at 5 years) (39). This cohort represents a selected subset of patients diagnosed with IBC with low disease burden. Case selection is likely to have a bearing on several other reported series where a breast conservation approach was used $(40,41)$. In large retrospective series the outcome after a conservative approach appears inferior, although again the caveats of retrospective studies are acknowledged (42). In sum, all authors agreed that classic, unequivocal IBC should be 
managed by mastectomy irrespective of response. The role of breast conservation remains controversial. A breast conservation approach could be tested in the context of carefully selected and followed patients on prospective clinical trials. Patients considered for or requesting breast conservation must be counseled regarding the potential for uncontrolled loco-regional recurrence.

Table 5. Surgical management recommendations by institution

\begin{tabular}{llll}
\hline Institution & Breast & Nodes & Caveat \\
\hline MD Anderson & MRM & Axillary dissection & No BCS \\
Dana-Farber Cancer Institute & MRM & Axillary dissection & No BCS \\
Northwestern University & MRM & Axillary dissection & No BCS \\
University of Michigan & MRM & Axillary dissection & No BCS \\
Egypt & MRM & Axillary dissection & No BCS \\
Senegal & MRM & Axillary dissection & No BCS \\
United Kingdom & MRM & Axillary dissection & BCS in select \\
& & & cases \\
\hline
\end{tabular}

Abbreviations: MRM, modified radical mastectomy; BCS, breast-conserving surgery.

In addition, the recommendation for the use of sentinel lymph node dissection in patients with clinically node-positive disease treated with NST, based on the results of the American College of Surgeons Oncology Group Z1071 trial, should be given with caution in IBC patients because this patient population was actively excluded from the study (43, 44). The results of a prospective clinical trial demonstrating failure to map in $75 \%$ of IBC patients, highlighting the importance of level I and II axillary lymph node dissection were presented (45). There was agreement that contralateral prophylactic mastectomy should be deferred to ensure that complications related to this non-oncologic surgery do not delay radiotherapy administration.

\section{Radiation}

All participants agreed that post-mastectomy radiotherapy is warranted in all cases and that targets include the chest wall and supraclavicular, infraclavicular, and internal mammary chain nodes. Radiation should specifically focus on any atypical geographic disease seen in the supraclavicular and neck nodes based on upfront cross sectional imaging. Similarly, care should be taken to review medical photographs to provide generous coverage of all skin disease. Recurrence patterns along the medial scar warrant coverage extension into the contralateral breast if needed. Although multiple approaches may be feasible, most experts agreed that minimal recommendations are chest wall boost to $60 \mathrm{~Gy}$, targeting of all undissected upfront gross nodal disease, and use of bolus to obtain an adequate skin dose. Further escalation to 66 Gy twice per day, improved locoregional control for patients younger than 45 years who had positive margins and poor response to chemotherapy $(46,47)$. On the basis of this evidence, treatment in patients with a lack of response and who are relatively young may be further personalized and is standard at MDA. An outstanding 4-year local control rate of 95\% similar to that for non-IBC patients, using the MDA tailored approach of accelerated dose escalation for high-risk non metastatic patients was recently published and presented, when a protocol of aggressive skin resection to negative margins, and complete axillary dissection, along with complete trimodal therapy, was utilized (48). In the setting of stage IV disease, radiotherapy was endorsed, using the same dose for local control.

Table 6: MDRing responses for surgery in inflammatory breast cancer (IBC)

\begin{tabular}{ll}
\hline Question & Answers \\
\hline All of the following could diminish the likelihood of & $20 \%$ answered breast-conserving surgery \\
achieving local control in patients with IBC EXCEPT: & $10 \%$ answered delaying or omitting surgery because skin biopsy remains positive after chemotherapy \\
& $52 \%$ answered including the internal mammary and supraclavicular lymph nodes in radiation fields \\
& $5 \%$ answered skin-sparing mastectomy \\
& $13 \%$ answered tissue expander placed after mastectomy \\
& $71 \%$ answered yes \\
I believe there is a role for sentinel lymph node mapping & $29 \%$ answered no \\
in patients with IBC & $13 \%$ answered yes \\
I would consider breast conservation for IBC & $87 \%$ answered no \\
& $73 \%$ answered yes \\
I would consider mastectomy with stage IV IBC & $27 \%$ answered no \\
& $13 \%$ answered yes \\
I would offer contralateral prophylactic mastectomy at & $87 \%$ answered no \\
the time of the primary surgical therapy for the affected & \\
breast for a patient with IBC & $16 \%$ answered yes \\
Skin-sparing mastectomy is appropriate for IBC & $84 \%$ answered no \\
\end{tabular}


Table 7. Radiotherapy management recommendations by institution

\begin{tabular}{|c|c|c|c|}
\hline Institution & Targets & Dose & Boost \\
\hline MD Anderson & Chest wall, ICV, SCV, IMN & $\begin{array}{l}50 \text { Gy; } 51 \text { Gy twice daily if }<45 \text { years, } \\
\text { no pathologic complete response, and } \\
\text { close margins }\end{array}$ & $\begin{array}{l}\text { Chest wall and involved undissected } \\
\text { upfront regional nodes: } 16 \text { Gy or } 15 \mathrm{~Gy} \\
\text { if twice per day }\end{array}$ \\
\hline Dana-Farber Cancer Institute & Chest wall, ICV, SCV, IMN & $50 \mathrm{~Gy}$ & $10 \mathrm{~Gy}$ \\
\hline
\end{tabular}

Abbreviations: ICV, infraclavicular; SCV, supraclavicular; IMN, internal mammary node.

Table 8: MDRing responses for radiotherapy in inflammatory breast cancer (IBC)

\begin{tabular}{ll}
\hline Question & Answers \\
\hline What approaches are well described to improve local control for patients with & $8 \%$ answered bolus to promote brisk erythema \\
IBC with an increased risk of local recurrence? & $23 \%$ answered dose $>60$ Gy \\
& $13 \%$ answered twice per day, accelerated regimens \\
& $56 \%$ answered all of the above \\
Which patients with IBC may benefit from radiation dose escalation or & $5 \%$ answered close or positive margins \\
acceleration? & $2 \%$ answered younger than 45 years \\
& $8 \%$ answered significant pathologic residual disease after systemic therapy \\
\hline
\end{tabular}

Table 9: MDRing responses for reconstruction in inflammatory breast cancer (IBC)

Questions

I believe it is appropriate to perform immediate reconstruction for IBC

I would recommend placing tissue expanders at initial mastectomy to allow implant-based reconstruction in IBC
Answers

$18 \%$ answered yes

$82 \%$ answered no

$27 \%$ answered yes

$73 \%$ answered no

\section{Reconstruction}

All agreed that delayed reconstruction should be considered in IBC. The importance of removing involved skin in IBC was reiterated, highlighting the consensus that there is no role for tissue expander-based immediate reconstruction, given that this preserves skin in a skin-involved cancer. Immediate reconstruction was also noted to be suboptimal given the requirement for post-mastectomy radiotherapy and the likelihood of the radiotherapy leading to the detriment of the immediate reconstruction cosmesis, as well as the difficulty imposed by the reconstruction in providing adequate dose to the at-risk internal mammary nodes and deep chest wall. Data summarizing the feasibility of delayed autologous tissue reconstruction in heavily irradiated IBC patients from MD Anderson was presented, showing success rates greater than 95\% $(49,50)$. Surgical options for lymphedema were reviewed, including lymphovenous bypass and vascularized lymph node transfer; $87 \%$ of patients have reported subjective improvements $(51,52)$.

\section{Conclusions}

In summary, by international expert consensus of the conference participants, the ideal IBC clinical framework includes upfront bilateral breast and nodal basin imaging with MRI and PET/CT when possible and upfront medical photographs. The treatment sequence is systemic chemotherapy for HER-2 normal and chemotherapy and dual antiHER-2 therapy for HER-2 positive subtypes, followed by modified radical mastectomy that affords surgical resection of the skin, breast, and axillary nodes when maximal disease response occurs. Skin-sparing mastectomy, immediate reconstruction, sentinel lymph node biopsy, and contralateral prophylactic mastectomy are not indicated. Post-mastectomy radiotherapy to the chest wall and undissected draining lymphatics, including internal mammary chain and supraclavicular nodes and including boost fields to the chest wall and any involved regional nodes at presentation, is uniformly appropriate. Endocrine therapy is appropriate after post-mastectomy radiotherapy. Delayed reconstruction (6 months to 1 year) is endorsed given improving contemporary local control rates and demonstrated feasibility. Lymphedema microsurgery is a reasonable consideration in this time frame.

There were areas of non-consensus that were not felt to be contentious. Broad variation in specific NST regimens highlights developing evidence in the non-IBC setting for the use of carboplatin in triple-negative breast cancer, as well as the role of dual HER2-neu targeting therapy, the use of dose-dense regimens, and timing of doxorubicin-containing regimens. In general, most participants continue to endorse the use of doxorubicin and taxane containing regimens. 
Radiotherapy regimens also varied in the settings in which these data could be reviewed, although targets and approach were unified. Discussion remains about the use of ovarian suppression with tamoxifen or aromatase inhibitors in premenopausal women, and there was no consensus on the use of adjuvant systemic therapy for cases of inadequate response.

The primary area of contentious non-consensus was in the use of skin-sparing surgeries such as breast conservation in carefully selected patients. Although the United Kingdom consensus statement has removed mastectomy as a mandatory surgery, strong dissention was voiced against considering breast conservation as an option for patients with, by definition, global skin-involved cancers and high rates of local progression. This may be an area of interest for trial development in motivated, well-informed patients.

Finally, new discussions for future work to optimize the staging system were introduced. Epidemiology data have clearly demonstrated that patients without erythema who present with consistent IBC features such as a rapid onset of global breast swelling, peau d'orange, and nipple retraction are correctly diagnosed with IBC, and the American Joint Committee on Cancer staging system should acknowledge that explicit erythema may not be evident in all IBC patients.

While non-scientific, the response rates of physician participants reflected the consensus of the experts overall. Areas of non-consensus may reflect misunderstanding or assertion of preference and are worthy of further study.

\section{Abbreviations}

IBC: inflammatory breast cancer; MDA: MD Anderson; NST: neoadjuvant systemic therapy; MRI: magnetic resonance imaging; PET: positron emission tomography; CT: computed tomography; CNB: core needle biopsy; $\mathrm{T}$ : paclitaxel; $\mathrm{P}$ : pertuzumab; $\mathrm{H}$ : trastuzumab; TCHP: taxane carboplatin trastuzumab pertuzumab; AC: doxorubicin cyclophosphamide; pCR: pathologic complete response; ER: estrogen receptor; LHRH: luteinizing hormone-releasing agonists; AI: aromatase inhibitors; BSC: breast-conserving surgery; MRM: modified radical mastectomy; ICV: infraclavicular; SCV: supraclavicular; IMN: internal mammary node.

\section{Acknowledgements}

Grant Support; NTU; Morgan Welch Inflammatory Breast Cancer Research Program and State of Texas Rare and Aggressive Breast Cancer Research Program Grant.

\section{Competing Interests}

The authors have declared that no competing interest exists.

\section{References}

1. Lu J, Hou J, Liu KY, Parmar S, De La Fuente A, Andersson B, et al. Asia-Pacific Hematology Consortium Report on approach to multiple myeloma. Survey results from the 6th International Hematologic Malignancies Conference: Bridging the Gap 2015, Beijing, China. Leuk Lymphoma. 2016;57:1534-8.

2. Liu K, Malhotra P, Parmar S, Wong R, Kornblau S, Mathews V, et al. Approach to AML Treatment. Survey Results from the 6th International Hematologic Malignancies Conference: Bridging the gap 2015,Beijing,China. J Leuk. 2015;3:186.

3. Kerbauy LN, Parmar S, Kutner JM, Gusmao BM, Hamerschlak N. Hematological approaches to multiple myeloma: trends from a Brazilian subset of hematologists. A cross-sectional study. Sao Paulo Med J. 2016;134:335-41.

4. Yamauchi H, Woodward WA, Valero V, Alvarez RH, Lucci A, Buchholz TA, et al. Inflammatory breast cancer: what we know and what we need to learn. Oncologist. 2012;17:891-9.

5. Yang WT, Le-Petross HT, Macapinlac H, Carkaci S, Gonzalez-Angulo AM, Dawood S, et al. Inflammatory breast cancer: PET/CT, MRI, mammography, and sonography findings. Breast Cancer Res Treat. 2008;109:417-26.

6. Günhan-Bilgen I, Üstün EE, Memiş A. Inflammatory Breast Carcinoma: Mammographic, Ultrasonographic, Clinical, and Pathologic Findings in 142 Cases. Radiology. 2002;223:829-38.

7. Schairer C, Brown LM, Mai PL. Inflammatory breast cancer: high risk of contralateral breast cancer compared to comparably staged non-inflammatory breast cancer. Breast Cancer Res Treat. 2011;129:117-24.

8. Chow CK. Imaging in inflammatory breast carcinoma. Breast Dis. 2005;22:45-54.

9. Le-Petross H, Uppendahl L, Stafford J. Sonographic features of inflammatory breast cancer. Semin Roentgenol. 2011;46:275-9.

10. Abeywardhana DY, Nascimento VC, Dissanayake D, Taylor D, Metcalf C, Saunders C, et al. Review of ultrasound appearance in inflammatory breast cancer: A pictorial essay. J Med Imaging Radiat Oncol . 2016;60:83-7.

11. Le-Petross HT, Cristofanilli M, Carkaci S, Krishnamurthy S, Jackson EF, Harrell RK, et al. MRI features of inflammatory breast cancer. AJR Am J Roentgenol. 2011;197:W769-76.

12. Iacconi C, Galman L, Zheng J, Sacchini V, Sutton EJ, Dershaw D, et al. Multicentric Cancer Detected at Breast MR Imaging and Not at Mammography: Important or Not? Radiology. 2016;279:378-84.

13. Liberman L. Breast MR imaging in assessing extent of disease. Magn Reson Imaging Clin N Am. 2006;14:339-49, vi.

14. Groheux D, Giacchetti S, Delord M, Hindie E, Vercellino L, Cuvier C, et al. 18F-FDG PET/CT in staging patients with locally advanced or inflammatory breast cancer: comparison to conventional staging. J Nucl Med. 2013;54:5-11.

15. Carkaci S, Sherman CT, Ozkan E, Adrada BE, Wei W, Rohren EM, et al. (18)F-FDG PET/CT predicts survival in patients with inflammatory breast cancer undergoing neoadjuvant chemotherapy. Eur J Nucl Med Mol Imaging. 2013;40:1809-16.

16. Niikura N, Odisio BC, Tokuda Y, Symmans FW, Hortobagyi GN, Ueno NT. Latest biopsy approach for suspected metastases in patients with breast cancer. Nat Rev Clin Oncol. 2013;10:711-9.

17. Walker GV, Niikura N, Yang W, Rohren E, Valero V, Woodward WA, et al. Pretreatment staging positron emission tomography/computed tomography in patients with inflammatory breast cancer influences radiation treatment field designs. Int J Radiat Oncol Biol Phys. 2012;83:1381-6.

18. Jacene H, DiPiro P, Bellon J, Nakhlis F, Hirshfield-Bartek J, Yeh E, et al. Abstract P5-02-01: Discrepancy between CT and FDG-PET/CT in the staging of patients with inflammatory breast cancer: Implications for radiation therapy treatment planning. Cancer Res. 2012;72:P5-02-1-P5--1.

19. Dawood S, Merajver SD, Viens P, Vermeulen PB, Swain SM, Buchholz TA, et al. International expert panel on inflammatory breast cancer: consensus statement for standardized diagnosis and treatment. Ann Oncol. 2011;22:515-23.

20. Bonnier P, Charpin C, Lejeune C, Romain S, Tubiana N, Beedassy B, et al. Inflammatory carcinomas of the breast: a clinical, pathological, or a clinical and pathological definition? Int J Cancer. 1995;62:382-5.

21. Raghav K, French JT, Ueno NT, Lei X, Krishnamurthy S, Reuben JM, et al. Inflammatory Breast Cancer: A Distinct Clinicopathological Entity Transcending Histological Distinction. PLoS One. 2016;11:e0145534.

22. Tomlinson JS, Alpaugh ML, Barsky SH. An intact overexpressed E-cadherin/alpha,beta-catenin axis characterizes the lymphovascular emboli of inflammatory breast carcinoma. Cancer Res. 2001;61:5231-41.

23. Loi S, Symmans WF, Bartlett JM, Fumagalli D, Van't Veer L, Forbes JF, et al. Proposals for uniform collection of biospecimens from neoadjuvant breast cancer clinical trials: timing and specimen types. Lancet Oncol. 2011;12:1162-8.

24. Dobbs J, Krishnamurthy S, Kyrish M, Benveniste AP, Yang W, Richards-Kortum R. Confocal fluorescence microscopy for rapid evaluation of 
invasive tumor cellularity of inflammatory breast carcinoma core needle biopsies. Breast Cancer Res Treat. 2015;149:303-10.

25. Untch M, Möbus V, Kuhn W, Muck BR, Thomssen C, Bauerfeind I, et al. Intensive Dose-Dense Compared With Conventionally Scheduled Preoperative Chemotherapy for High-Risk Primary Breast Cancer. J Clin Oncol. 2009;27:2938-45.

26. Ditsch N, Vodermaier A, Hinke A, Burghardt S, Lenhard M, Lohrs B, et al. Dose-dense intensified sequential versus conventionally-dosed anthracycline and taxane-containing neoadjuvant therapy in patients with inflammatory breast cancer. Anticancer Res. 2012;32:3539-45.

27. Ellis GK, Barlow WE, Gralow JR, Hortobagyi GN, Russell CA, Royce ME, et al. Phase III comparison of standard doxorubicin and cyclophosphamide versus weekly doxorubicin and daily oral cyclophosphamide plus granulocyte colony-stimulating factor as neoadjuvant therapy for inflammatory and locally advanced breast cancer: SWOG 0012. J Clin Oncol. 2011;29:1014-21.

28. Ueno NT, Buzdar AU, Singletary SE, Ames FC, McNeese MD, Holmes FA, et al. Combined-modality treatment of inflammatory breast carcinoma: twenty years of experience at M. D. Anderson Cancer Center. Cancer Chemother Pharmacol. 1997;40:321-9.

29. Gianni L, Eiermann W, Semiglazov V, Lluch A, Tjulandin S, Zambetti M, et al. Neoadjuvant and adjuvant trastuzumab in patients with HER2-positive locally advanced breast cancer $(\mathrm{NOAH})$ : follow-up of a randomised controlled superiority trial with a parallel HER2-negative cohort. Lancet Oncol. 2014;15:640-7.

30. Gianni L, Pienkowski T, Im Y-H, Roman L, Tseng L-M, Liu M-C, et al. Efficacy and safety of neoadjuvant pertuzumab and trastuzumab in women with locally advanced, inflammatory, or early HER2-positive breast cancer (NeoSphere): a randomised multicentre, open-label, phase 2 trial. Lancet Oncol. 2012;13:25-32

31. Schneeweiss A, Chia S, Hickish T, Harvey V, Eniu A, Hegg R, et al. Pertuzumab plus trastuzumab in combination with standard neoadjuvant anthracycline-containing and anthracycline-free chemotherapy regimens in patients with HER2-positive early breast cancer: a randomized phase II cardiac safety study (TRYPHAENA). Ann Oncol. 2013.

32. von Minckwitz G, Procter M, de Azambuja E, Zardavas D, Benyunes M, Viale G, et al. Adjuvant Pertuzumab and Trastuzumab in Early HER2-Positive Breast Cancer. N Engl J Med. 2017.

33. Francis PA, Regan MM, Fleming GF, Láng I, Ciruelos E, Bellet M, et al. Adjuvant Ovarian Suppression in Premenopausal Breast Cancer. N Engl J Med. 2015;372:436-46.

34. Sikov WM, Berry DA, Perou CM, Singh B, Cirrincione CT, Tolaney SM, et al. Impact of the Addition of Carboplatin and/or Bevacizumab to Neoadjuvant Once-per-Week Paclitaxel Followed by Dose-Dense Doxorubicin and Cyclophosphamide on Pathologic Complete Response Rates in Stage II to III Triple-Negative Breast Cancer: CALGB 40603 (Alliance). J Clin Oncol. 2015;33:13-21.

35. von Minckwitz G, Schneeweiss A, Loibl S, Salat C, Denkert C, Rezai M, et al. Neoadjuvant carboplatin in patients with triple-negative and HER2-positive early breast cancer (GeparSixto; GBG 66): a randomised phase 2 trial. Lancet Oncol. 2014;15:747-56.

36. Masuda N, Lee SJ, Ohtani S, Im YH, Lee ES, Yokota I, et al. Adjuvant Capecitabine for Breast Cancer after Preoperative Chemotherapy. N Engl J Med. 2017;376(22):2147-2159.

37. Low JA, Berman AW, Steinberg SM, Danforth DN, Lippman ME, Swain SM. Long-term follow-up for locally advanced and inflammatory breast cancer patients treated with multimodality therapy. J Clin Oncol. 2004;22:4067-74.

38. Rea D, Francis A, Hanby AM, Speirs V, Rakha E, Shaaban A, et al. Inflammatory breast cancer: time to standardise diagnosis assessment and management, and for the joining of forces to facilitate effective research. Br J Cancer. 2015;112:1613-5.

39. Brzezinska M, Williams LJ, Thomas J, Michael Dixon J. Outcomes of patients with inflammatory breast cancer treated by breast-conserving surgery. Breast Cancer Res Treat. 2016;160:387-91.

40. Bonev V, Evangelista M, Chen JH, Su MY, Lane K, Mehta R, et al. Long-term follow-up of breast-conserving therapy in patients with inflammatory breast cancer treated with neoadjuvant chemotherapy. Am Surg. 2014:80:940-3.

41. Chen H, Wu K, Wang M, Wang F, Zhang M, Zhang P. A standard mastectomy should not be the only recommended breast surgical treatment for non-metastatic inflammatory breast cancer: A large population-based study in the Surveillance, Epidemiology, and End Results database 18. Breast. 2017;35:48-54.

42. Dawood S, Lei X, Dent R, Gupta S, Sirohi B, Cortes J, et al. Survival of women with inflammatory breast cancer: a large population-based study. Ann Oncol. 2014;25:1143-51.

43. Boughey JC, Suman VJ, Mittendorf EA, Ahrendt GM, Wilke LG, Taback B, et al. Sentinel lymph node surgery after neoadjuvant chemotherapy in patients with node-positive breast cancer: the ACOSOG Z1071 (Alliance) clinical trial. JAMA. 2013;310:1455-61.

44. Stearns V, Ewing CA, Slack R, Penannen MF, Hayes DF, Tsangaris TN Sentinel lymphadenectomy after neoadjuvant chemotherapy for breast cancer may reliably represent the axilla except for inflammatory breast cancer. Ann Surg Oncol. 2002;9:235-42.

45. DeSnyder SM, Mittendorf EA, Le-Petross C, Krishnamurthy S, Whitman GI, Ueno NT, et al. Prospective Feasibility Trial of Sentinel Lymph Node Biopsy in the Setting of Inflammatory Breast Cancer. Clin Breast Cancer. 2017.
46. Woodward WA. Postmastectomy Radiation Therapy for Inflammatory Breast Cancer: Is More Better? Int J Radiat Oncol Biol Phys. 2014;89:1004-5.

47. Bristol IJ, Woodward WA, Strom EA, Cristofanilli M, Domain D, Singletary $\mathrm{SE}$, et al. Locoregional treatment outcomes after multimodality management of inflammatory breast cancer. Int J Radiat Oncol Biol Phys. 2008;72:474-84.

48. Rosso KJ, Tadros AB, Weiss A, Warneke CL, DeSnyder S, Kuerer H, et al. Improved Locoregional Control in a Contemporary Cohort of Nonmetastatic Inflammatory Breast Cancer Patients Undergoing Surgery. Ann Surg Oncol. 2017.

49. Chang EI, Chang EI, Ito R, Zhang H, Nguyen AT, Skoracki RJ, et al. Challenging a traditional paradigm: 12-year experience with autologous free flap breast reconstruction for inflammatory breast cancer. Plast Reconstr Surg. 2015;135:262e-9e.

50. Chang EI, Chang EI, Soto-Miranda MA, Zhang H, Nosrati N, Crosby MA, et al. Comprehensive Evaluation of Risk Factors and Management of Impending Flap Loss in 2138 Breast Free Flaps. Ann Plast Surg. 2016;77:67-71.

51. Scaglioni MF, Arvanitakis M, Chen YC, Giovanoli P, Chia-Shen Yang J, Chang EI. Comprehensive review of vascularized lymph node transfers for lymphedema: Outcomes and complications. Microsurgery. 2016.

52. Nguyen AT, Chang EI, Suami H, Chang DW. An algorithmic approach to simultaneous vascularized lymph node transfer with microvascular breast reconstruction. Ann Surg Oncol. 2015;22:2919-24. 